\title{
J-INTEGRAL AND CRACK GROWTH ANALYSIS OF 2-D ELEMENT
}

\author{
Anurag Jain ${ }^{1}$, Dr Pankaj Agarwal ${ }^{2}$ and Dr. S.K. Dhakad ${ }^{3}$
}

Abstract- Crack growth could be encountered in many components, which can be made of either thin or thick steel plate and therefore, be subjected to a stress and strain condition respectively. The load applied in a sold body containing a crack will include a yield zone near its tips with a dimensions that will depend on the mechanical properties of the material as well as the crack length. The paper presents, J- integral function, the evaluation of J-integral value was carried out for the different crack lengths and using finite element method for the evaluation of J-integral by ANSYS software.

Keywords - J-integral, Crack, FEA, FCA,E, etc.

\section{INTRODUCTION}

The crack propagation plays an important role in the fracture process and at the crack tip stress intensity factors are carried and to be known and correlated with crack growth rate, order to define, the time to time inspection is required if a damage tolerance design was chose to be applied during the in-service lifetime a component.

In this work, a 2-d geometry was analyzed through FEM method to evaluate the J-integral value at a Different crack length. For the evaluation of J-integral value ANSYS software is used.

\section{J-INTEGRAL}

The J-integral represents a way to calculate the strain energy release rate, or work (energy) per unit fracture surface area, in a material. ${ }^{[1]}$ The theoretical concept of J-integral was developed in 1967 by Cherepanov ${ }^{[2]}$ and in 1968 by Jim Rice ${ }^{[3]}$ independently, who showed that an energetic contour path integral (called $J$ ) was independent of the path around a crack.

Methods were developed, which allowed measurement of critical fracture properties using laboratory-scale specimens for materials in which sample sizes are too small and for which the assumptions of Linear Elastic Fracture Mechanics (LEFM) do not hold, ${ }^{[6]}$ and to infer a critical value of fracture energy $J_{\text {Ic }}$. The quantity $J_{\text {Ic }}$ defines the point at which large-scale plastic yielding during propagation takes place under mode one loading. ${ }^{[1][4]}$

The J-integral is equal to the strain energy release rate for a crack in a body subjected to monotonic loading. ${ }^{[5]}$ This is generally true, under quasistatic conditions, only for linear elastic materials. For materials that experience small-scale yielding at the crack tip, $J$ can be used to compute the energy release rate under special circumstances such as monotonic loading in mode III (antiplane shear). The strain energy release rate can also be computed from $J$ for pure power-law hardening plastic materials that undergo small-scale yielding at the crack tip.

The quantity $J$ is not path-independent for monotonic mode I and mode II loading of elastic-plastic materials, so only a contour very close to the crack tip gives the energy release rate. Also, Rice showed that $J$ is path-independent in plastic materials when there is no non-proportional loading. Unloading is a special case of this, but non-proportional plastic loading also invalidates the path-independence. Such non-proportional loading is the reason for the path-dependence for the in-plane loading modes on elastic-plastic materials.

\section{METHODOLOGY}

A finite element method (abbreviated as FEM) is a numerical technique to obtain an approximate solution to a class of problems governed by elliptic partial differential equations. Such problems are called as boundary value problems as they consist of a partial differential equation and the boundary conditions. The finite element method converts the elliptic partial differential equation into a set of algebraic equations which are easy to solve. The initial value problems which consist of a parabolic or hyperbolic differential equation and the initial conditions (besides the boundary conditions) can not be completely solved by the finite element method. The parabolic or hyperbolic differential equations contain the time as one of the independent variables. To convert the time or temporal derivatives into algebraic expressions, another numerical technique like the finite difference method (FDM) is required. Thus, to solve an initial value problem, one needs both the

\footnotetext{
${ }^{1}$ M. Tech, Samrat Ashok Technological Institute, Vidisha M.P.

${ }^{2}$ H.O.D. Mechanical Department, Samrat Ashok Technological Institute, Vidisha M.P

${ }^{3}$ Assistant Professor Mech. Dept. Samrat Ashok Technological Institute, Vidisha M.P
} 
finite element method as well as the finite difference method where the spatial derivatives are converted into algebraic expressions by FEM and the temporal derivatives are converted into algebraic equations by FDM.

The finite element method involves the following steps.

1-First, the governing differential equation of the problem is converted into an integral form. These are two techniques to achieve this : (i) Variational Technique and (ii) Weighted Residual Technique. In variational technique, the calculus of variation is used to obtain the integral form corresponding to the given differential equation. This integral needs to be minimized to obtain the solution of the problem. For structural mechanics problems, the integral form turns out to be the expression for the total potential energy of the structure. In weighted residual technique, the integral form is constructed as a weighted integral of the governing differential equation where the weight functions are known and arbitrary except that they satisfy certain boundary conditions. To reduce the continuity requirement of the solution, this integral form is often modified using the divergence theorem. This integral form is set to zero to obtain the solution of the problem. For structural mechanics problems, if the weight function is considered as the virtual displacement, then the integral form becomes the expression of the virtual work of the structure.

2-In the second step, the domain of the problem is divided into a number of parts, called as elements. For one-dimensional (1D) problems, the elements are nothing but line segments having only length and no shape. For problems of higher dimensions, the elements have both the shape and size. For two-dimensional (2D) or axi-symmetric problems, the elements used are triangles, rectangles and quadrilateral having straight or curved boundaries. Curved sided elements are good choice when the domain boundary is curved. For three-dimensional (3-D) problems, the shapes used are tetrahedron and parallelepiped having straight or curved surfaces. Division of the domain into elements is called a mesh.

3-In this step, over a typical element, a suitable approximation is chosen for the primary variable of the problem using interpolation functions (also called as shape functions) and the unknown values of the primary variable at some pre-selected points of the element, called as the nodes. Usually polynomials are chosen as the shape functions. For 1-D elements, there are at least 2 nodes placed at the end-points. Additional nodes are placed in the interior of the element. For 2-D and 3-D elements, the nodes are placed at the vertices (minimum 3 nodes for triangles, minimum 4 nodes for rectangles, quadrilaterals and tetrahedral and minimum 8 nodes for parallelepiped shaped elements). Additional nodes are placed either on the boundaries or in the interior. The values of the primary variable at the nodes are called as the degrees of freedom.

To get the exact solution, the expression for the primary variable must contain a complete set of polynomials (i.e., infinite terms) or if it contains only the finite number of terms, then the number of elements must be infinite. In either case, it results into an infinite set of algebraic equations. To make the problem tractable, only a finite number of elements and an expression with only finite number of terms are used. Then, we get only an approximate solution. (Therefore, the expression for the primary variable chosen to obtain an approximate solution is called an approximation). The accuracy of the approximate solution, however, can be improved either by increasing the number of terms in the approximation or the number of elements.

4-In the fourth step, the approximation for the primary variable is substituted into the integral form. If the integral form is of variational type, it is minimized to get the algebraic equations for the unknown nodal values of the primary variable. If the integral form is of the weighted residual type, it is set to zero to obtain the algebraic equations. In each case, the algebraic equations are obtained element wise first (called as the element equations) and then they are assembled over all the elements to obtain the algebraic equations for the whole domain (called as the global equations).

5-In this step, the algebraic equations are modified to take care of the boundary conditions on the primary variable. The modified algebraic equations are solved to find the nodal values of the primary variable.

6-In the last step, the post-processing of the solution is done. That is, first the secondary variables of the problem are calculated from the solution. Then, the nodal values of the primary and secondary variables are used to construct their graphical variation over the domain either in the form of graphs (for 1-D problems) or 2-D/3-D contours as the case may be.

\section{EXPERIMENT AND RESULT}

In order to calculate the fracture mechanics parameter such as J-integral a specimen was designed according to ASTM standard and simulation were carried out .

A model was designed in ANSYS mechanical software and every model is run on ANSYS to obtain the fracture property i.e. J-integral. 


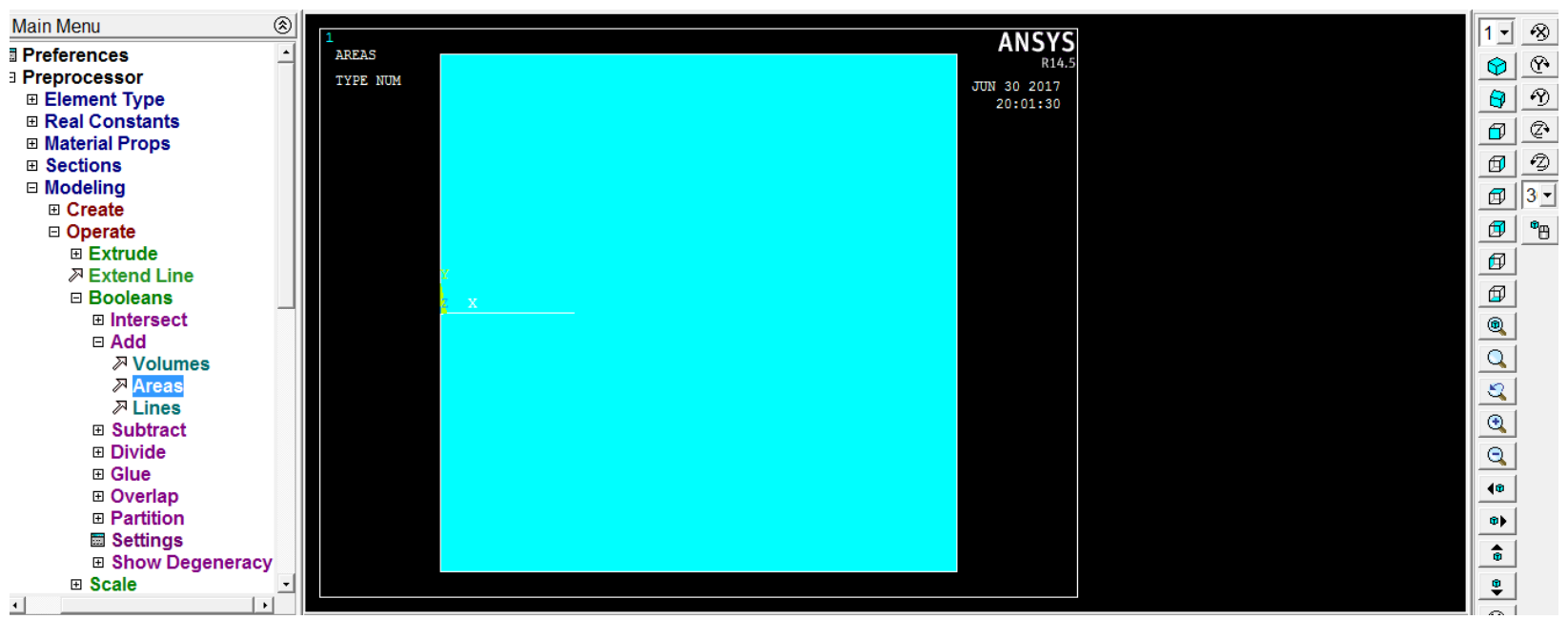

Fig. 1 The fig 1 shows the model design in the ANSYS. Which is having a fine crack.

Eile Select List Plot PlotCtrls WorkPlane Parameters Macro MenuCtrls Help

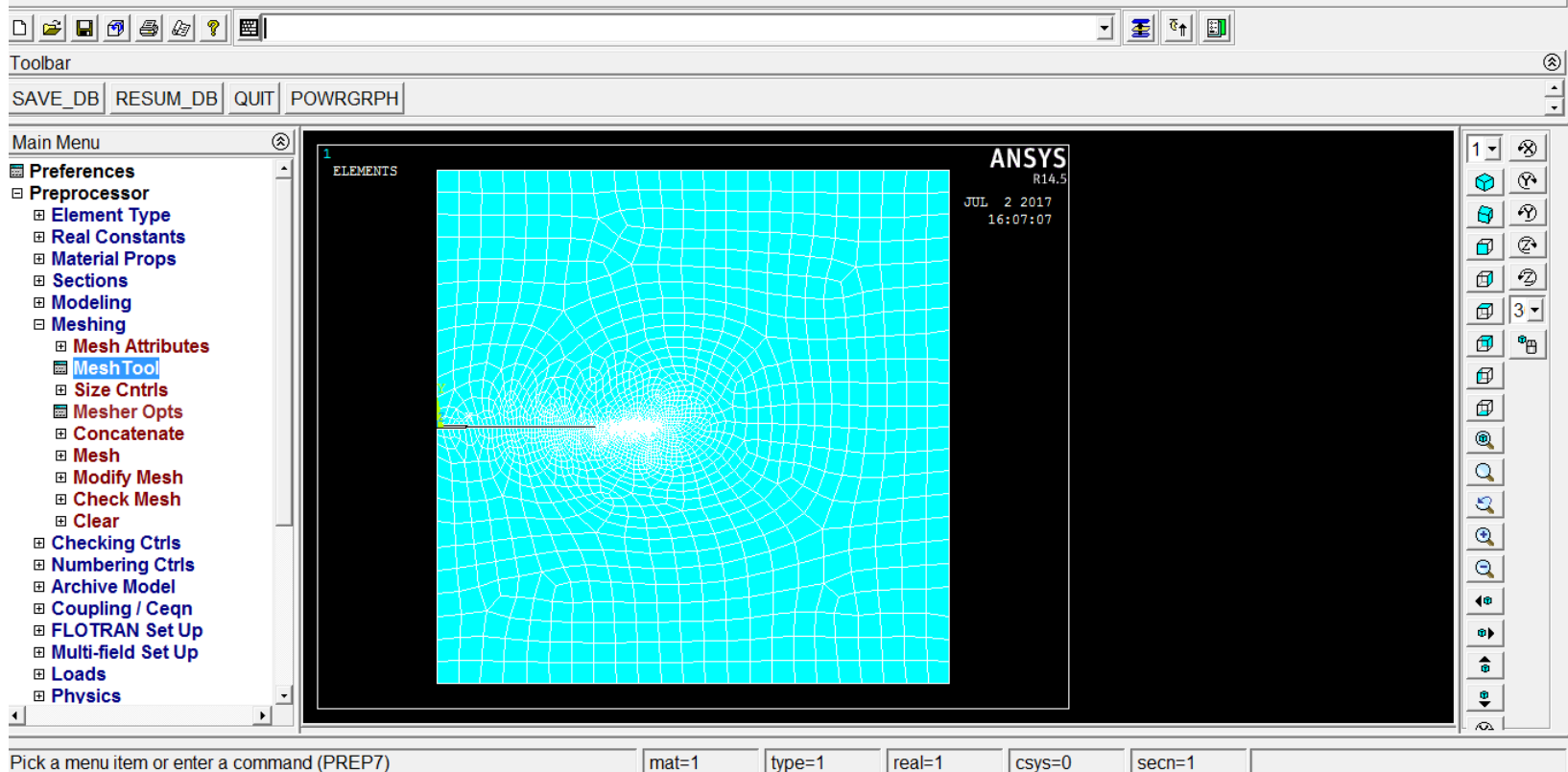

Fig 2 The fig 2 shows the messed model of the specimen which having a hexagonal free meshing.

\begin{tabular}{|l|l|l|}
\hline s. no & Crack length & J-integral \\
\hline 1 & $11.7 \mathrm{~mm}$ & 0.0545 \\
\hline 2 & $13 \mathrm{~mm}$ & 0.0668 \\
\hline 3 & $14.3 \mathrm{~mm}$ & 0.085 \\
\hline
\end{tabular}

Table $1 \mathrm{~J}$-integral values

Table 1 showing the J-integral value at the different crank length which coming over the software.

Table2 ASTM J-integral

\begin{tabular}{|l|l|l|}
\hline S.no & crack & J-integral \\
\hline 1 & $11.7 \mathrm{~mm}$ & 0.05 \\
\hline 2 & $13 \mathrm{~mm}$ & 0.068 \\
\hline 3 & $14.3 \mathrm{~mm}$ & 0.094 \\
\hline
\end{tabular}




\section{CONCLUSION}

From the literature review which has been done so far and the experiments performed on the test specimen it has been seen that the results given by the software follows the same rendition as the ASTM j-integral. In future prospects we will try to validate the results on different material and geometry using the FEA method.

\section{REFERENCES}

[1] Van Vliet, Krystyn J. (2006); "3.032 Mechanical Behavior of Materials"

[2] G. P. Cherepanov, The propagation of cracks in a continuous medium, Journal of Applied Mathematics and Mechanics, 31(3), 1967, pp. 503-512

[3] J. R. Rice, A Path Independent Integral and the Approximate Analysis of Strain Concentration by Notches and Cracks, Journal of Applied Mechanics, 35, 1968, pp. 379-386.

[4] Lee, R. F., \& Donovan, J. A. (1987). J-integral and crack opening displacement as crack initiation criteria in natural rubber in pure shear and tensile specimens. Rubber chemistry and technology, 60(4), 674-688

[5] Meyers and Chawla (1999): "Mechanical Behavior of Materials," 445-448.

[6] Yoda, M., 1980, The J-integral fracture toughness for Mode II, Int. J. of Fracture, 16(4), pp. R175-R178

[7] Brocks,W, Cornec,A, Scheider,I, 2002. Computational Aspects of Nonlinear Fracture Mechanics. Tech. Note GKSS/WMS/02/05 internal report.

[8] Hellan, Kåre, 1985. Introduction to fracture mechanics. In: Eichberg M. (Ed.). International Student Edition, Singapore, pp. 22

[9] Budiansky, B., Rice, J. R., 1973. Conservation Laws and Energy-Release Rates. Journal of Applied Mechanics, 40, pp. $201-03$.

[10] Rigby, R. H., Aliabadi, M. H., 1998. Decomposition of the Mixed-Mode J-Integral - Revisited. Int. J. Solids Structures, 35 , pp. $2073-2099$.

[11] Hutchinson, JW, 1983. Fundamentals of the Phenomenological Theory of Nonlinear Fracture Mechanics. Journal of App. Mech, 50, 1042-51.

[12] E 399 - 90 (Reapproved 1997). Standard Test Method for Plane-Strain Fracture Toughness of Metallic Materials. ASTM International.

[13] E647-00. Standard Test Method for Measurement of Fatigue Crack Growth Rates. ASTM International. 